\title{
ESTADO DA ARTE DA PESQUISA REALIZADA EM PROGRAMAS DE PÓS-GRADUAÇÃO EM ENSINO DE CIÊNCIAS E MATEMÁTICA NO BRASIL
}

\author{
STATE OF THE ART IN THE GRADUATE PROGRAMS IN TEACHING SCIENCE AND MATHEMATICS IN BRAZIL
}

\author{
Antonella Carvalho de Oliveira, Damaris Godoy Leite, Antonio Carlos Frasson, Luiz \\ Alberto Pilatti*
}

Universidade Tecnológica Federal do Paraná - Campus Ponta Grossa

Rua Monterio Lobato, s/n - Jardim Carvalho. CEP: 84000-000

Ponta Grossa, PR - Brasil - Telefone: (+42) 3220-4800

E-mail: antonella_0611@hotmail.com,damaris@cescage.edu.br, ancafras@gmail.com, lapilatti@utfpr.edu.br

\begin{abstract}
Resumo
O trabalho apresenta o estado da arte da pesquisa em Ensino de Ciências e Matemática no Brasil, na forma de Dissertações de Mestrado Profissional, Dissertações de Mestrado Acadêmico e Teses de Doutorado, defendidas em programas de pós-graduação stricto sensu recomendados pela CAPES nos anos de 2010 a 2013. Trata-se de uma pesquisa qualitativa exploratória, cuja análise documental pauta-se em procedimentos eminentemente descritivos. A pesquisa abrangeu 11 programas de pós-graduação Mestrado Acadêmico, 16 Mestrado Profissional e sete Doutorados. Foram localizados 368 trabalhos, sendo 186 dissertações de mestrado acadêmico, 154 dissertações de mestrado profissional e 28 teses de doutorado. Os aspectos analisados foram: ano de defesa; região; nível de ensino abordado; disciplina contemplada no trabalho e produtos gerados pelas pesquisas. A importância do trabalho deve-se ao fato de que o mesmo pode ser utilizado como instrumento para analisar as temáticas e foco das pesquisas realizadas nos programas de Ensino de Ciências e Matemática no Brasil, contribuindo para a consolidação do campo, podendo, inclusive, orientar novas pesquisas na área.
\end{abstract}

Palavras-chave: ensino de ciências e matemática; produção acadêmica brasileira, estado da arte.

\begin{abstract}
This research analyzes the state of art in Teaching Mathematics and Science researches in Brazil, in the form of Professional Master Degree Dissertations, Academic Dissertations and Doctoral Thesis, defended in post-graduate programs recommended by CAPES in the years 2010-2013. It is a qualitative exploratory research which approach was based on a descriptive perspective. The survey covered 11 programs of graduate Academic Masters, 16 Professional and seven Doctoral Dissertations. 368 papers were found, being 186 academic Master's dissertations, 154 professional master's dissertations and 28 doctoral thesis. The aspects analyzed in the research were: year of defense; region; level of education addressed; discipline at work and contemplated products generated by the research. The importance of the work in question is due to the fact that it can be used as a tool to analyze the themes and focus of research conducted in the Teaching of Science and Mathematics programs in Brazil, contributing to the consolidation of the field. It can also be used asa guiide further research in the area.
\end{abstract}

Keywords: teaching of science and mathematics; brazilian academic production, state of the art 


\section{INTRODUÇÃO}

No período Colonial não houve preocupação com o ensino de ciências e matemática no Brasil, somente na segunda metade do século XVIII José Fernandes Pinto Alpoim, engenheiro militar, publicou o primeiro livro de matemática no Brasil. Já o ensino de ciências iniciou apenas no século XX, tendo um expoente crescimento após a segunda Guerra Mundial (D'AMBROSIO, 1999). As alterações ocorridas na sociedade pós-guerra, crescente industrialização e expansão do desenvolvimento científico e tecnológico, acabaram por impactar o currículo escolar, pois havia nesse momento uma demanda reprimida por "novos cientistas" (KRASILCHIK, 2000). Prova disso foi a criação, em 1955, do Instituto de Matemática Pura e Aplicada (IMPA), sendo a primeira unidade de pesquisa criada pelo Conselho Nacional de Pesquisas (CNPq), um ano após a própria criação deste. A ideia de que o desenvolvimento científico e tecnológico leva ao desenvolvimento social e igualitário passa a ser questionada e em decorrência surgem as dúvidas que giram em torno de um ensino de ciências que contemple as implicações sociais daquele.

A Organização das Nações Unidas para a Educação, a Ciência e a Cultura (UNESCO) lança, nos anos 80, a meta "o acesso ao conhecimento científico" sob o lema "Ciência para Todos". Posteriormente na década de 90 o Brasil publica no campo pedagógico a Lei de Diretrizes e Bases da Educação Nacional (LDBEN/1996), os Parâmetros Curriculares Nacionais (PCNs/1997) e o Sistema Nacional de Avaliação (SNA). Fato é que no último século as descobertas nas diversas áreas do conhecimento humano abriram caminho para uma reflexão sobre o ensinar e o aprender. O acesso à escola para todos, fruto das reformas educacionais da década de 90 , bem como outros mecanismos de acesso à informação, possibilitaram que as pessoas hoje sejam mais conscientes dos seus direitos, assim a expectativa desses frente à escola foi alterada e suscitam questões como: Qual o papel da escola nos dias atuais? A escola hoje serve para quê?

Para Friedrich e Benite (2010), a escola parece hoje cumprir, mesmo que precariamente, apenas a garantia de certificação da conclusão de determinado grau de ensino. Nesta perspectiva instaurase a função reparadora da educação, como dívida social, que denota a restauração de um direito negado: uma escola de qualidade. Por essa razão se fazem necessárias pesquisas de revisão de literatura, especialmente nas áreas de educação e ensino. Determinados os objetivos e a 
problemática do trabalho, a seguir apresenta-se a descrição da metodologia empregada na presente pesquisa.

\section{PERCURSO METODOLÓGICO}

O procedimento adotado para apresentar o estado da arte, cujo corpus é constituído de dissertações de mestrado profissional e acadêmico, bem como teses de doutorado, com foco no Ensino de Ciências e Matemática foi análise documental. Para Ludke e André (2012, p. 39) documentos podem se constituir em "uma fonte poderosa de onde podem ser retiradas evidências que fundamentem afirmações e declarações do pesquisador, representam ainda uma fonte natural de informação". A análise documental não é somente uma fonte de informação escrita, mas brota em um estipulado contexto fornecendo informações sobre esse mesmo contexto, além de servir perfeitamente ao propósito de pesquisas de cunho exploratório.

As fases para o desenvolvimento da pesquisa foram adaptadas da dissertação de Mestrado em Educação Científica e Tecnológica de Mário Cézar Amorim de Oliveira (2011) da Universidade Federal de Santa Catarina (UFSC):

- Fase 1) Busca e obtenção de todas as produções acadêmicas no Brasil, que foram identificadas a partir de programas recomendados pela CAPES com foco no ensino de ciências e matemática;

- Fase 2) Leitura e análise dos trabalhos;

- Fase 3) Configuração e classificação dos aspectos a serem analisados e

- Fase 4) Análise dos dados encontrados a partir dos aspectos elencados na fase 3.

Pela própria natureza da pesquisa essas fases nem sempre seguiram um padrão linear, alguns procedimentos ocorreram concomitantemente e outros foram sobrepostos, entretanto, as fases serão explicitadas na sequencia em que forem citadas.

A fase 1 consistiu em localizar os programas pertencentes a grande área "Multidisciplinar", área "Ensino", recomendados pela CAPES, com produções alocadas no Ensino de Ciências e Matemática. Foram selecionados 14 programas conforme o quadro 1. Além desses programas, constatamos a existência de mais 14 programas capazes de alocar pesquisas relacionadas ao Ensino de Ciências e Matemática, conforme o quadro 2. 
Quadro 1 - Programas de pós-graduação Stricto Sensu na área de Ensino de Ciências e Matemática.

\begin{tabular}{|c|c|c|c|c|c|}
\hline PROGRAMA & INSTITUIÇÃO & UF & $\begin{array}{l}\text { MESTRADO } \\
\text { ACADÊMICO }\end{array}$ & $\begin{array}{c}\text { MESTRADO } \\
\text { PROFISSIONAL }\end{array}$ & DOUTORADO \\
\hline Ensino de Ciências e Matemática & UFAC & $\mathrm{AC}$ & & $x$ & \\
\hline Ensino de Ciências e Matemática & UFAL & $\mathrm{AL}$ & & $\mathrm{x}$ & \\
\hline Ensino de Ciências e Matemática & UFAM & AM & $\mathrm{X}$ & & \\
\hline Ensino de Ciências e Matemática & UFC & $\mathrm{CE}$ & & $x$ & \\
\hline Ensino de Ciências e Matemática & UFU & MG & & $\mathrm{x}$ & \\
\hline Ensino de Ciências e Matemática & UEPB & PB & & $\mathrm{X}$ & \\
\hline Ensino de Ciências e Matemática & CEFET RJ & $\mathrm{RJ}$ & & $\mathrm{X}$ & \\
\hline Ensino de Ciências e Matemática & UFPEL & $\mathrm{RS}$ & & $\mathrm{X}$ & \\
\hline Ensino de Ciências e Matemática & UCS & RS & & $x$ & \\
\hline Ensino de Ciências e Matemática & UPF & RS & & $\mathrm{X}$ & \\
\hline Ensino de Ciências e Matemática & ULBRA & RS & $\mathrm{X}$ & & $\mathrm{X}$ \\
\hline Ensino de Ciências e Matemática & FUFSE & SE & $\mathrm{X}$ & & \\
\hline Ensino de Ciências e Matemática & UNICSUL & SP & & $x$ & \\
\hline Ensino de Ciências e Matemática & IFSP & SP & & $\mathrm{X}$ & \\
\hline
\end{tabular}

Fonte: Adaptado da Coordenação de Aperfeiçoamento de Pessoal em Nível Superior (2014).

Quadro 2 - Programas de pós-graduação stricto sensu que alocam pesquisas relacionadas ao Ensino de Ciências e Matemática.

\begin{tabular}{|c|c|c|c|c|c|}
\hline PROGRAMA & INSTITUIÇÃO & UF & $\begin{array}{l}\text { MESTRADO } \\
\text { ACADÊMICO }\end{array}$ & $\begin{array}{l}\text { MESTRADO } \\
\text { PROFISSIONAL }\end{array}$ & DOUTORADO \\
\hline Educação em Ciências e Matemática & IFES & ES & & $\mathrm{X}$ & \\
\hline Educação em Ciências e em Matemática & UFPR & PR & $\mathrm{x}$ & & \\
\hline Educação em Ciências e Matemática & UFG & GO & $\mathrm{x}$ & & \\
\hline Educação em Ciências e Matemática & PUCRS & RS & $\mathrm{x}$ & & $\mathrm{X}$ \\
\hline Educação em Ciências e Matemática & UFMT & MT & & & $\mathrm{X}$ \\
\hline Educação em Ciências e Matemática & UFPA & $\mathrm{PA}$ & $\mathrm{x}$ & & $\mathrm{X}$ \\
\hline Educação para a Ciência e a Matemática & UEM & $\mathrm{PR}$ & $\mathrm{x}$ & & $\mathrm{x}$ \\
\hline Educação para Ciências e Matemática & IFG & GO & & $\mathrm{X}$ & \\
\hline Ensino de Ciências e Educação Matemática & UEPB & PB & $\mathrm{x}$ & & \\
\hline Ensino de Ciências e Educação Matemática & UEL & PR & $\mathrm{x}$ & & $\mathrm{X}$ \\
\hline Ensino de Ciências Naturais e Matemática & UFRN & RN & & $\mathrm{X}$ & \\
\hline Ensino de Ciências Naturais e Matemática & UNICENTRO & PR & & $\mathrm{X}$ & \\
\hline Ensino de Ciências Naturais e Matemática & FURB & SC & & $\mathrm{X}$ & \\
\hline Multiunidades em Ensino de Ciências e Matemática & UNICAMP & SP & $x$ & & $x$ \\
\hline
\end{tabular}

Fonte: Adaptado da Coordenação de Aperfeiçoamento de Pessoal em Nível Superior (2014).

Consideramos, portanto, a produção de 11 programas de pós-graduação stricto sensu Mestrado Acadêmico, 16 programas de pós-graduação stricto sensu Mestrado Profissional e sete programas de pós-graduação stricto sensu Doutorado e um total de 368 trabalhos, sendo 186 dissertações de mestrado acadêmico, 154 dissertações de mestrado profissional e 28 teses de doutorado A maior limitação da pesquisa decorreu do foto de que alguns programas não divulgam em seu site os trabalhos resultantes das pesquisas realizadas. 
A fase 2 consistiu na leitura dos trabalhos concomitantemente com a fase 3 , que tinha como objetivo configurar e classificar os aspectos estabelecidos nos trabalhos. Foram definidos, a partir de Oliveira (2011), os seguintes aspectos, julgados relevantes, a serem mencionados no referido estado da arte: ano de defesa; região; nível de ensino abordado; disciplina contemplada no trabalho e produtos gerados pelas pesquisas. Para cada trabalho lido foi atribuída uma ficha descritiva individual para posterior tabulação. Na fase 4 construímos um banco de dados utilizando como ferramenta o software Excel, com vistas a facilitar a leitura das temáticas e o foco das pesquisas, possibilitando assim indicar possíveis hiatos e tendências na área.

\section{ANÁLISE DOS DADOS}

\subsection{Produção acadêmica no tempo}

A partir da tabela 1 verifica-se que nos anos de 2010 a 2013 houve aumento de trabalhos provenientes dos programas de mestrado acadêmico. No ano de 2011 foi registrado aumento de cinco trabalhos em relação a 2010, em 2012 houve acréscimo de quatro trabalhos em relação a 2011 e em 2013 três trabalhos foram publicados a mais do que 2012. O aumento do número de trabalhos deve-se, em parte, à abertura de novos programas de Mestrado Acadêmico no período estudado, como o Programa em Educação em Ciências e Matemática do Instituto Federal de Educação, Ciência e Tecnologia do Espírito Santo (IFES), que iniciou suas atividades em 2011.

Tabela 1 - Produção acadêmica em números absolutos entre os anos de 2010 e 2013.

\begin{tabular}{l|l|l|l|l|l}
\hline Produção Acadêmica & 2010 & 2011 & 2012 & 2013 & Total \\
\hline Dissertações Mestrado Acadêmico & 40 & 45 & 49 & 52 & 186 \\
\hline Dissertações Mestrado Profissional & 40 & 40 & 34 & 40 & 154 \\
\hline Teses & - & 6 & 5 & 17 & 28 \\
\hline Total Geral & & & & & 368 \\
\hline
\end{tabular}

Fonte: Da pesquisa (2014).

O número de trabalhos oriundos do mestrado profissional pouco oscilou durante o período avaliado, iniciou com 40 trabalhos em 2010, permaneceu estável em 2011, baixou para 34 produções em 2012 e voltou a 40 produções em 2013, não é possível afirmar com plenitude as razões para o resultado encontrado em 2012. No Brasil o número de programas em Mestrado Profissional (16 programas no total) excede o número de Mestrado Acadêmico (11 programas), 
assim o aumento no percentual de trabalhos provenientes do mestrado acadêmico em relação ao mestrado profissional é considerado significativo, pois, o mestrado acadêmico passa de 40 trabalhos em 2010 para 52 em 2013, enquanto o mestrado profissional mantem-se com 40 trabalhos em média durante o período pesquisado.

Dos sete programas que contemplam cursos de doutorado, só foi possível o acesso aos dados de dois programas, o que justifica o número de produções encontradas nesse item. No ano de 2013 houve um acréscimo significativo em teses de doutorado, qual seja, passou de seis trabalhos publicados em 2011 para 17 em 2013, lembrando que esses números referem-se ao programa de pós-graduação em Ensino de Ciências e Educação Matemática da Universidade Estadual de Londrina (UEL) e do programa em Ensino de Ciências e Matemática da Universidade Luterana do Brasil (ULBRA).

\subsection{Produção acadêmica por região brasileira}

De acordo com os dados apresentados na tabela 2, verificamos que a região Sul contribuiu com 29\% da produção (108 trabalhos), seguida pela região Nordeste, com $27 \%$ do total (98 trabalhos), a região Centro-Oeste contribuiu com 25\% (91 trabalhos) e a região Sudeste com 17\% (64 trabalhos). O programa em Educação em Ciências e Matemática stricto sensu doutorado da Universidade Federal do Pará (a primeira defesa de tese aconteceu em 2013), único da região Norte, não divulga os trabalhos na integra, somente o número de defesas realizadas.

Tabela 2 - Produção acadêmica por região brasileira.

\begin{tabular}{l|l|l|l|l|l}
\hline & Sul & Sudeste & Centro-Oeste & Norte & Nordeste \\
\hline Dissertações Mestrado Acadêmico & 79 & 02 & 91 & - & 14 \\
\hline Dissertações Mestrado Profissional & 8 & 62 & - & - & 84 \\
\hline Teses & 21 & - & - & 7 & - \\
\hline Total & 108 & 64 & 91 & 7 & 98 \\
\hline$\%$ & $29 \%$ & $17 \%$ & $25 \%$ & $2 \%$ & $27 \%$ \\
\hline
\end{tabular}

Fonte: Da pesquisa (2014). 
A região Sul abriga dez programas de pós-graduação stricto sensu na área pesquisada (cinco Programas de Mestrado Acadêmico, quatro programas de Mestrado Profissional e um programa de Doutorado), logo, a maior contribuição concentrada nessa região não é um fato inesperado. $A$ produção da região Nordeste também se destaca, uma vez que resulta de trabalhos desenvolvidos em quatro Programas de Pós-Graduação, distribuídos em diferentes estados: Alagoas, Sergipe, Paraíba e Rio Grande do Norte.

A região Centro-Oeste contou com a contribuição de apenas um Programa de Pós-Graduação, pois somente o programa de Mestrado Acadêmico em Educação em Ciências e Matemática da Universidade Federal de Goiás (UFG) divulga seus trabalhos. A UFG contribuiu com 91 dissertações de mestrado entre os anos de 2010 e 2013, demonstrando que a instituição tem uma participação expressiva no cenário nacional no que tange ao ensino de Ciências e Matemática se comparada a produção de outras regiões, que contam com um número maior de instituições que disponibilizam suas pesquisas. Embora a região Sudeste aloque sete programas de pós-graduação só foi possível localizar 64 trabalhos, distribuídos em dois programas.

\subsection{Produção acadêmica por nível de ensino abordado na pesquisa}

A caracterização quanto ao nível de ensino abordado na pesquisa usou a classificação do Ministério da Educação (MEC): Educação Básica e Ensino Superior. Considerando que a Educação Básica é dividida em Educação Infantil, Ensino Fundamental Fase I, Ensino Fundamental Fase II e Ensino Médio.

O número absoluto das dissertações e teses por nível de escolaridade e de acordo com o ano de defesa está representado na tabela 3. Houve um percentual de 19,22\% de trabalhos em que não foi possível localizar o nível de ensino contemplado, incluído neste percentual aproximadamente $5 \%$ de pesquisas cujo tema não está relacionado à área de ensino.

Do total de 368 trabalhos, 111 foram desenvolvidos visando ao Ensino Médio, 96 ao Ensino Superior, 29 à fase I do Ensino Fundamental, 56 à fase II do Ensino Fundamental, 02 à Educação Infantil e em 74 pesquisas não localizamos a informação. A ausência deste dado não significa que 
estes trabalhos não tenham relação com a área de ensino em ciências e matemática, pois verificamos que várias pesquisas discorrem sobre temas que podem abarcar todos os níveis de ensino, tais como avaliação, projeto político pedagógico, afetividade e outros.

Tabela 3 - Produção acadêmica por nível de ensino abordado na pesquisa.

\begin{tabular}{l|c|c|c|c|c|c}
\hline Ano & 2010 & 2011 & 2012 & 2013 & Total & $\%$ \\
\hline Ensino Superior & 20 & 31 & 20 & 25 & 96 & $26 \%$ \\
\hline Ensino Médio & 26 & 32 & 24 & 29 & 111 & $30 \%$ \\
\hline Ensino Fundamental - Fase II & 10 & 15 & 18 & 13 & 56 & $15 \%$ \\
\hline Ensino Fundamental - Fase I & 9 & 7 & 7 & 6 & 29 & $8 \%$ \\
\hline Educação Infantil & 1 & - & 1 & - & 02 & 0,5 \\
\hline Informação não localizada & 14 & 6 & 18 & 36 & 74 & $20 \%$ \\
\hline Total & & & & 368 & $100 \%$ \\
\hline
\end{tabular}

Fonte: Da pesquisa (2014).

Privilegiar o ensino médio como objeto de estudo indica que a preocupação dos pesquisadores vinculados a programas de pós-graduação está em consonância com o MEC que fomenta o Pacto Nacional pelo Fortalecimento do Ensino Médio visando a promover a formação continuada dos professores e coordenadores pedagógicos que atuam no Ensino Médio, em consonância com a LDBEN, com as DCNS para o Ensino Médio e com as Diretrizes Curriculares para a Educação Básica. Em contrapartida a educação infantil e a fase I tem participação inexpressiva neste cenário, contribuindo respectivamente com $0,5 \%$ e $8 \%$ dos trabalhos pesquisados. Mantendo a mesma linha de análise do ensino médio e em função do Pacto Nacional para Alfabetização na Idade Certa, consideramos que os programas de pós-graduação stricto sensu estudados não abarcam toda a necessidade, em todos os níveis de ensino, de pesquisas que o Brasil exige. Outro fator a considerar é a quantidade de trabalhos semelhantes por orientador, determinados orientadores só trabalham com um nível de ensino e em um caso especial localizamos um programa aonde $65 \%$ dos trabalhos focam o ensino superior.

\subsection{Produção acadêmica por disciplina contemplada na pesquisa.}

A Tabela 4 apresenta a produção acadêmica de acordo com a disciplina abordada no trabalho. A disciplina de matemática figura com $51,5 \%$ dos trabalhos, sendo que aproximadamente $45 \%$ deste percentual remetem as possibilidades de ensino-aprendizagem de forma mais eficaz. Em seguida 
aparece Física com 15\%, Biologia e Ciências representam respectivamente 8\% e 9\%, Química 6,5\% e em $10 \%$ dos casos não identificamos a disciplina, provavelmente em função de produções focadas em estudos que envolvem o processo ensino-aprendizagem como um todo e não em uma disciplina específica.

Dentro dessas produções que envolvem questões pedagógicas, salientamos a presença de trabalhos que abrangem questões pontuais como: avaliação, currículo, tecnologias aplicadas à educação e a formação inicial do professor. Entretanto, faltam trabalhos com temas como indisciplina, violência na escola, educação especial, diversidade e demais temas relacionados ao ensino.

Tabela 4 - Produção acadêmica por disciplina contemplada na pesquisa.

\begin{tabular}{l|l|l|l|l|l|l}
\hline & 2010 & 2011 & 2012 & 2013 & Total & $\%$ \\
\hline Biologia & 06 & 07 & 07 & 09 & 29 & $8 \%$ \\
\hline Ciências & 03 & 13 & 05 & 12 & 33 & $9 \%$ \\
\hline Física & 11 & 11 & 15 & 18 & 55 & $15 \%$ \\
\hline Matemática & 47 & 49 & 42 & 52 & 190 & $51,5 \%$ \\
\hline Química & 06 & 07 & 02 & 09 & 24 & 37 \\
\hline Informação não localizada & 07 & 04 & 17 & 09 & 368 & $10 \%$ \\
\hline Total & & & & & $100 \%$ \\
\hline
\end{tabular}

Fonte: Da pesquisa (2014).

Apesar de questões como indisciplina ou violência na escola não terem aparecido no conjunto das obras analisadas, encontramos trabalhos que traçam o perfil dos alunos e suas dificuldades de aprendizagem, também encontramos dois trabalhos que tratam da evasão escolar. $\mathrm{O}$ viés mais explorado nas disciplinas é a formação inicial do professor, o que leva a duas vertentes de raciocínio: 1) Pode representar que os pesquisadores entendem que, de certa forma, o fracasso escolar está associado ao despreparo docente, provavelmente fruto da sua formação inicial, ou; 2) Pode representar que os pesquisadores pretendem desmitificar essa visão neoliberal na educação. A disciplina de matemática aparece em $50,12 \%$ dos casos, sendo que em aproximadamente $28 \%$ deste percentual aparece vinculada a programas de mestrado profissional, índice parecido acontece com as outras disciplinas, tendo ficado reservado ao mestrado acadêmico e doutorado obras de cunho pedagógico de modo geral e não focadas especificamente em uma disciplina. Em 
função da necessidade de produzir um produto no mestrado profissional, a fim de resolver questões pontuais da realidade escolar, justifica-se a produção encontrada.

\subsection{Produção acadêmica por produto desenvolvido.}

Houve dificuldade em mapear os produtos originados das dissertações e teses, em grande parte devido à falta de clareza nos objetivos dos referidos trabalhos. Ainda assim detectamos que 57 trabalhos apresentam como produto "Materiais Didáticos", que vão desde sequências didáticas até manuais para conselho de classe e demais instâncias colegiadas. Em segundo lugar, mas com grande diferença, aparece o "Jogo Didático", possível de identificar em seis dissertações de mestrado profissional, porém em quatro trabalhos não é demonstrado como os jogos foram validados e/ou não comprovaram que o jogo atende a necessidade apresentada na pesquisa. 0 produto Objeto de Aprendizagem apareceu em cinco pesquisas. O Blog, Histórias em Quadrinhos (HQ) e Instrumento de Avaliação figuram três vezes entre as produções elencadas. Oficina Pedagógica e Charge empataram como produto criado em uma única pesquisa.

Tabela 5 - Produção acadêmica por produto desenvolvido.

\begin{tabular}{l|l|l|l|l|l|l}
\hline & 2010 & 2011 & 2012 & 2013 & Total & $\%$ \\
\hline Blog & - & 1 & 2 & - & 3 & 0,8 \\
\hline Charge & - & - & - & 1 & 1 & 0,2 \\
\hline HQ & - & 1 & 1 & 1 & 3 & 0,8 \\
\hline Instrumento de Avaliação & - & 2 & - & 1 & 3 & 0,8 \\
\hline Jogo Pedagógico & 1 & 3 & 1 & 1 & 6 & 1,6 \\
\hline Materiais Didáticos & 5 & 14 & 17 & 21 & 57 & 15,4 \\
\hline Objeto de Aprendizagem & 1 & - & 4 & - & 5 & 1,3 \\
\hline Oficina Pedagógica & - & - & - & 1 & 1 & 0,2 \\
\hline Informação não localizada & 73 & 70 & 63 & 83 & 289 & 78,5 \\
\hline Total & & & & 368 & $100 \%$ \\
\hline
\end{tabular}

Fonte: Da pesquisa (2014).

O que chama a atenção é o alto índice de trabalhos que não apresentam produtos, $79,48 \%$ do total das pesquisas. Em programas de mestrado profissional não foi detectado produto em $20 \%$ dos casos, ou seja, aproximadamente 30 trabalhos do total divulgado no período analisado não apresentou o resultado final da pesquisa em forma de produto. 
Convém ressaltar também que alguns produtos se esgotam em si, como dois dos blogs visitados que estão desatualizados. Possivelmente o projeto tenha sido abandonado pelos pesquisadores. A charge e as histórias em quadrinhos são ferramentas pedagógicas interessantes, no entanto, não houve produção em escala, sendo que cada escola, campo de trabalho, ficou com um número ínfimo de exemplares, o que na prática acaba inviabilizando o trabalho com esse recurso.

\section{CONSIDERAÇÕES FINAIS}

De acordo com a pesquisa exploratória realizada, o objetivo do trabalho ora apresentado foi alcançado, pois conseguimos empreender o estado de arte acerca do ensino de ciências e matemática no Brasil a partir dos programas de pós-graduação stricto sensu. Somam-se a esse fato algumas questões pontuais que serão socializadas.

O número de programas de pós-graduação stricto sensu mestrado sobrepõem-se, em quantidade, aos programas de doutorado, sendo 16 programas de mestrado profissional, 11 de mestrado acadêmico e sete de doutorado. Tendo em vista que é prática adotada pela CAPES e avalizada pelas IES a consolidação dos programas de mestrado para a posterior implantação dos cursos de doutorado, os dados encontrados estão no padrão esperado. Quanto ao número de trabalhos, localizamos 186 trabalhos oriundos do mestrado acadêmico, 154 do mestrado profissional e 45 do doutorado. Reforçando que esses números não expressam necessariamente a realidade, pois, muitos programas de pós-graduação não divulgam os resultados de suas produções.

É flagrante o número de trabalhos com escopo na formação inicial e continuada de professores. Em que pese o inegável progresso da área, os desafios neste campo exigem permanente reavaliação, pois as pesquisas apontam que é necessário desenvolver ferramentas, mecanismos, estratégias para aperfeiçoar essa formação, possibilitando que as novas gerações de professores sejam efetivamente melhor preparadas. Nesse sentido a escassez crônica de recursos humanos qualificados e a qualidade insuficiente da educação básica são compartilhadas pelos pesquisadores. Há também uma tentativa em achar caminhos para estimular a pesquisa entre os alunos em todos os níveis de ensino. 
Embora seja grande o número de eventos divulgados em todas as partes do país com os temas indisciplina, violência na escola, educação especial, diversidade, sentimos que há necessidade de maior robustez, melhor rendimento por parte dos programas de mestrado e doutorado em pesquisar tais assuntos, ou seja, existe um hiato na produção desses temas vinculados à área de ciências e matemática e provavelmente pesquisas consistentes com esses temas têm muito a contribuir para com a área em questão.

As pesquisas mostram claramente a distinção que o ensino médio ocupa nos trabalhos oriundos de programas de pós-graduação stricto sensu de todo o Brasil. Isso remete à necessidade premente de manter investigações também em outros níveis de ensino, especialmente na educação infantil e no ensino fundamental.

\section{REFERÊNCIAS}

BRASIL, Lei de Diretrizes e Bases para a Educação Nacional. Lei no 9.394, de 20 de dezembro de 1996. Diário Oficial da União. Ministério da Educação: Brasília, 1996.

CAPES. COORDENAÇÃO DE APERFEIÇOAMENTO DE PESSOAL DE NÍVEL SUPERIOR. Disponível em http://www.capes.gov.br. Acesso em 05 de julho de 2014.

D’AMBRÓSIO, U. História da Matemática no Brasil: Uma Visão Panorâmica até 1950. Saber Y Tiempo, vol. 2, $\mathrm{n}^{\circ}$ 8, jul/dez. p. 7-37. 1999.

FRIEDRICH, M.; BENITE, C. R. M. Uma abordagem sobre o ensino de ciências e matemática no programa nacional de inclusão de jovens: do discurso à prática. Revista Brasileira de Pesquisa em Educação e Ciências. Vol. 10, no 3, p. 101-124, 2010.

KRASILCHIK, M. Reformas e realidade: o caso do ensino das ciências. Perspectiva, v. 14, n. 1, p. 85-93, 2000.

LUDKE, M.; André, M. E. D. A. Pesquisa em educação: abordagens qualitativas. São Paulo, E.P.U., 2012.

OLIVEIRA, M. C. A. de. Aspectos da pesquisa acadêmica brasileira sobre o ensino dos temas "Origem da Vida" e "Evolução Biológica". Dissertação (Mestrado em Educação Científica e Tecnológica). Universidade Federal de Santa Catarina. Florianópolis, 2011. 